\title{
Eicosapentaenoic Acid as Adjuvant for Cachexia in Cancer's Patients
}

\author{
Soeseno Hadi, ${ }^{1}$ Cynthia Kurniawan, ${ }^{2}$ Jansen Budiono ${ }^{2}$ \\ ${ }^{1}$ Department of Pathology Anatomy, Faculty of Medicine, Universitas Padjdajaran-Dr. Hasan Sadikin General
Hospital \\ ${ }^{2}$ Faculty of Medicine, Universitas Padjdajaran-Dr. Hasan Sadikin General Hospital
}

\begin{abstract}
Received:

March 4, 2014

Revised:

Cachexia is a frequent problem in cancer patients associated with mortality and morbidity since it causes death, reduced therapy effectiveness, as well as decreased quality of life. Cachexia emerges from interactions of several factors, namely metabolic effect of cancer cells, factors released by cancer cells, cytokines release from host cell in response to tumors, and side effects of therapies. Combination of these factors contributes to anorexia, decreased body fat and muscle, and weight loss. Unfortunately, there is no gold standard for treatment of cachexia. Several studies found that eicosapentaenoic acid (EPA) might improve cancer cachexia. It has potential blocking effects on several cachexia underlying factors. It may reverse weight loss, increase lean body mass, and improve the efficacy of therapy and the quality of life. The objective of this literature review is to describe and give basic understanding of EPA as adjuvant for cancer cachexia.
\end{abstract}

June 5, 2014

Accepted:

November 1, 2014

Keywords: Cachexia, cancer, EPA

IJIHS. 2015;3(1):1-6

\section{Introduction}

Cachexia is one of the most frequent problems found in cancer patients that represents a state of progressive wasting of body fat and muscle which is often accompanied by anorexia. ${ }^{1}$ This problem occurs in $50 \%$ of cancer patients. ${ }^{2}$

Cachexia can be the cause of death in cancer patients. Degree of weight loss in cachexia may be used as a prognostic factor; the higher the extent of weight loss, the shorter the patient's survival time.

Cancer patient's condition becomes worse with cachexia because it reduces the patient's tolerance to the toxic effect of therapies. The chemotherapy dosages need to be adjusted in these patients, thus decreasing the treatment effectiveness. Cachexia also has an association with fatigue and poor physical performance, presumably, which then lead to a decrease in the quality of life. Patients become increasingly dependent due to the fact that they have lost their capacity to function normally. ${ }^{1,3}$

\footnotetext{
Correspondence:

Soeseno Hadi, Department of Pathology Anatomy,

Faculty of Medicine, Universitas Padjdajaran-Dr. Hasan Sadikin General Hospital

Jl. Pasteur No. 38, Bandung, Indonesia

e-mail: senoyang@ymail.com
}

Several interventions for treating cachexia have shown limited benefits. ${ }^{4}$ The application of hypercaloric feeding has not succeeded in reversing weight loss. Appetite stimulants, such as megestrol acetate, increase appetite but do not result in weight gain. ${ }^{5}$ Raising caloric intake is not sufficient to manage cachexia.

Later studies found eicosapentaenoic acid (EPA) may improve cancer cachexia. ${ }^{6}$ It may block several underlying factors of cachexia. The objective of this review is to describe and provide basic understanding of EPA as adjuvant for cancer cachexia. This review will benefit future research by providing deeper knowledge for effectiveness of EPA in treating cancer cachexia.

\section{Cancer Cachexia}

Cancer cachexia is a multi-factorial syndrome defined by an ongoing loss of skeletal muscle mass (with or without loss of fat mass) that cannot be fully reversed by using conventional nutritional supports and leads to progressive functional impairment. The pathophysiology of this condition is characterized by a negative protein and energy balance, which is driven by a variable combination of reduced food intake 
and abnormal metabolism. ${ }^{7}$ The occurence of cachexia depends on the cancer type. Cachexia develops more commonly in certain types of cancer (Table 1). ${ }^{1}$

Cancer cachexia progresses in three stages, which create a continuum. Precachexia, as the first stage, is characterized by a weight loss of less than $5 \%$ and is sometimes accompanied by metabolic and endocrine abnormalities. This stage is followed by Cachexia, which is a stage when the weight loss is more than $5 \%$ with systemic inflammation and reduced food intake. The last stage is refractory cachexia, in which there is severe muscle wasting, low performance score, and immune suppression. Prognosis of this stage is poor with expected survival of less than 3 months. ${ }^{6}$ The refractory cachexia can be diagnosed using the following criteria: meet criteria for cachexia; prognosis of less than 3 months; World Health Organization performance status of 3 or 4; unresponsive to anti-cancer therapy; ongoing catabolism at increasing rate; and unsuitable for artificial nutritional support.?

A model to assess signs and symptoms of cachexia has been proposed. In this model, four aspects need to be considered: Storage, which assesses the difference between usual to current body weight, velocity of weight loss, weight loss corrected for fluid retention or obesity, and signs of other nutritional deficiency; Intake, which assesses anorexia, satiety, nausea, vomiting, disturbances of taste or smelling, other gastrointestinal symptoms, percentage of normal intake, and dietary diary for 1-2 days; Potential, which assesses tumor [catabolic] activity, C-reactive protein; and Performance, which assesses the performance status, cachexia-related suffering, and the prognosis. $^{7}$

\section{Pathophysiology of Cancer Cachexia}

Cachexia emerges from interactions of several factors: cancer cells' metabolic activity, soluble factors that are produced by cancer cells, host's response, and side effects of therapies. This study shows chematic mechanism underlying cachexia (Fig. 1).

One of the most prominent features of cachexia is weight loss. The weight loss results from an energy imbalance. Negative energy balance in the cachexia is a product of increased energy expenditure due to inflammation and decreased energy intake caused by anorexia.

Cancer cells need higher energy to support their excessive proliferation. However, their metabolic processes are ineffective. These cells need more glucose than the normal cells to generate same amount of adenosine triphosphate (ATP). Cancer cells use glycolysis as the main metabolic activity, a process known as the Warburg effect. It has been suggested that the mutation of Deoxyribonucleic Acid (DNA)that leads to mitochondrial dysfunction prevents cancer cells to use the krebs cycle metabolic pathway. Pyruvate from glycolysis turns into lactate. The accumulated lactate is then transferred to the liver in which 2 mol of lactate is converted to $1 \mathrm{~mol}$ of glucose using $6 \mathrm{~mol}$ of ATP. This process is called Cori cycle. ${ }^{1}$

Table 1 Cancer in which Cachexia Develops ${ }^{3}$

\begin{tabular}{lc}
\hline \multicolumn{1}{c}{ Malignancy } & $\begin{array}{c}\text { Patients with } \\
\text { Cachexia (\%) }\end{array}$ \\
\hline Gastric cancer & 85 \\
Pancreatic cancer & 83 \\
Non-small cell lung cancer & 61 \\
Small cell lung cancer & 57 \\
Prostate cancer & 56 \\
Colon cancer & 54 \\
Unfavourable non-Hodgkin's & 48 \\
lymphoma & 40 \\
Sarcoma & 39 \\
Acute non-lymphocytic & 36 \\
leukaemia & 31 \\
Breast cancer & \\
Favourable non-Hodgkin's & \\
lymphoma &
\end{tabular}

In response to cancer, host cells produce some soluble factors, such as tumor necrosis factor- $\alpha$ (TNF- $\alpha$ ) and interleukins (ILs). These cytokines mediate the inflammatory activities. Acute phase response (APR) is a process that normally occurs in inflammatory responses. In APR, liver cells shift production of albumin to C-reactive protein (C-RP). During a normal inflammatory reaction, APR gives beneficial effects. However, in cancer, prolonged APR increases resting energy expenditure (REE), thus accelerates weight loss. ${ }^{3,9,10}$

Anorexia is a spontaneous, unintended decline in food intake. ${ }^{1}$ In this condition, the loss of appetite is caused by the combination of the biochemical and psychological factors. The appetite biochemical regulation depends 
on the activity of neuropeptide-Y (NP-Y) and proopiomelanocortin (POMC). These factors present in neurons of hypothalamic arcuate nucleus. Neuropeptide-Y is the main appetite stimulant, while POMC opposes its action. Serotonin and corticotropin releasing factor (CRF), which levels are increased by proinflammatory cytokine, depress the activity of NP-Y. These cytokines cause anorexia through their effects in brain nucleus and modulation of neurons appetite regulation. ${ }^{5}$

Psychological factors contribute to anorexia as well. They involve an interplay between the behavioral, emotional, and also the perceptual responses. Examples of behavioral responses are nausea and vomiting, which may cause patients to avoid eating. Emotional responses, such as fear, depression, and anxiety, which usually are associated with cancer patients, also reduce appetite. Perceptual responses such as beliefs about food may also influence eating habit. ${ }^{11}$

Energy imbalance is not the only possible cause of cachexia. Cytokines and other factors produced by tumor evoke cachexia by directly stimulating proteins and fat breakdown. The proteolysis inducing factors (PIF) and lipid mobilizing factors (LMF) are soluble factors that are produced by tumor.

Pro-inflammatory cytokines along with the PIF cause skeletal muscle breakdown. They act through the IKB kinase (IKK)

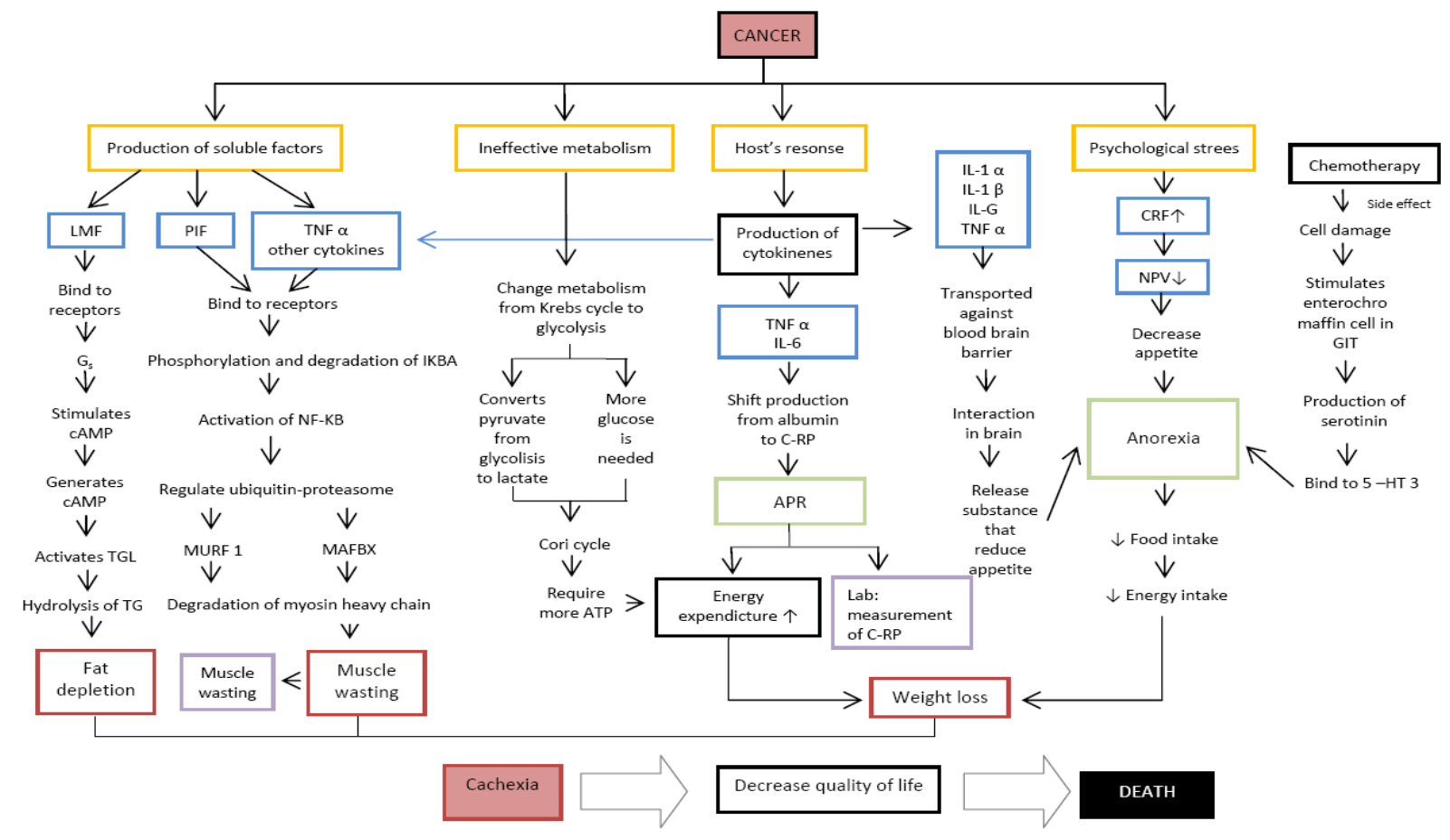

Fig. 1 Cachexia Schematic Mechanism. Abbreviation: AC = adenylate cyclase; APR = acute phase response; $A T P=$ adenosine triphosphate; cAMP = cyclic adenosine monophosphate; $\mathrm{CRF}=$ corticotropin-releasing factor; $\mathrm{C}-\mathrm{RP}$ = C-reactive protein; DNA = deoxyribonucleic acid; GIT = gastrointestinal track; IкBA = inhibitor kappa-B $\alpha$; IL = interleukin; LMF = lipid mobilizing factors; MAFBX = muscle atrophy F-box; MURF 1 = muscle RING finger-1; NF$\kappa \mathrm{B}=$ nuclear factor kappa-B; NPY = neuropeptide-Y; proteolysis-inducing factors; $\mathrm{TG}=$ tiglyceride; $\mathrm{TGL}=$ triglyceride lipase; $\mathrm{TNF}-\alpha=$ tumor necrosis factor- $\alpha$; 5 -HT 3 = serotonin receptor 3 . 


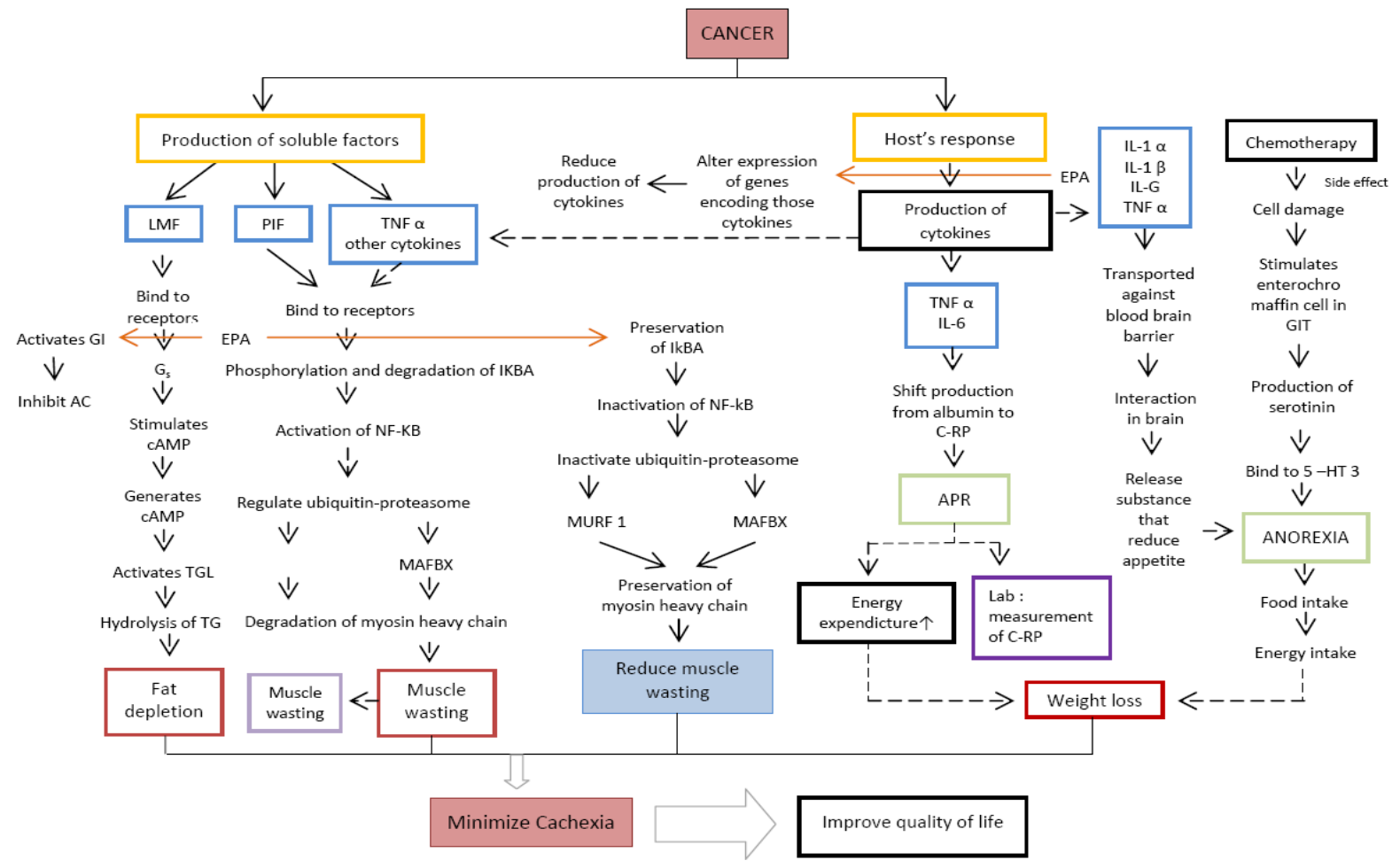

Fig. 2 Schematic Effect of EPA as Adjuvant for Cancer Cachexia. Abbreviation: $\mathrm{AC}=$ adenylate cyclase; $\mathrm{APR}=$ acute phase response; $\mathrm{ATP}=$ adenosine triphosphate; $c A M P=$ cyclic adenosine monophosphate; $\mathrm{CRF}$ = corcotropinreleasing factor; $\mathrm{C}-\mathrm{RP}=\mathrm{C}$-reactive protein; $\mathrm{DNA}=$ deoxyribonucleic acid; GIT = gastrointestinal track; IкBA = inhibitor kappa-B $\alpha$; IL = interleukin; LMF = lipid mobilizing factors; MAFBX = muscle atrophy F-box; MURF 1 = muscle RING finger-1; NF- $\mathrm{KB}$ = nuclear factor kappa-B; NPY = neuropeptide-Y; proteolysis-inducing factors; TG = tiglyceride; TGL = triglyceride lipase; TNF- $\alpha=$ tumor necrosis factor- $\alpha$; 5 -HT $3=$ serotonim receptor 3.

complex. This complex phosphorylates and degrades inhibitor kappa-B-alpha (IкB $\alpha$ ). The degradation of $І \kappa \mathrm{B} \alpha$ permits the activation of nuclear factor kappa-B (NF- $\kappa \mathrm{B})$ to regulate the ubiquitin proteasome activity (Fig. 1). The activation of this pathway produces two muscle-specific ubiquitin ligases, muscle RING finger-1 (MuRF1) and muscle atrophy F-box (MAFBx). These factors induce muscle loss due to proteolysis of myosin heavy chain. ${ }^{2}$

Lipid mobilizing factors stimulate lipolysis through action of triglyceride lipase. It binds to $\beta 3$-adrenergic receptor, a G-protein receptor. $\mathrm{G}$-protein receptor consists of three subunits: $\alpha, \beta$, and $\gamma$. The activity of G-protein depends on its $\alpha$ subunit. It has Gs and Gi pathways, which activate and inhibit adenylate cyclase, respectively. Adenylate cyclase is an enzyme that catalyzes the formation of cyclic adenosine monophosphate or cAMP from the ATP. Binding of LMF to its receptor activates adenylate cyclase which, in turns, induces the formation of cAMP, causing the phosphorylation and activation of triglyceride lipase. This enzyme then hydrolyzes triglyceride into fatty acids and glycerol. In addition to the mechanism described above, the fat mobilization is also stimulated by TNF- $\alpha$. Tumor necrosis factor- $\alpha$ induces lipolysis through mitogen activated protein kinase (MEK). ${ }^{1}$ 
Cancer patients are usually treated by using chemotherapy. Chemotherapy triggers adverse effect to normal cells in the body. As a response to the cellular damages causes by chemotherapy, the enterochromaffin cells in the gastrointestinal tract release serotonin. Serotonin binds the serotonim receptors (5HT3) in the gastrointestinal tract and the generated impulses are sent to the vomiting center in brain. ${ }^{12}$ Vomiting may contribute to cachexia by decreasing the appetite and nutrient intake. ${ }^{11}$

\section{Eicosapentaenoic Acid}

Eicosapentaenoic acid or omega-3 fatty acid is a long chain polyunsaturated fatty acid. Its natural source is fish oil. However, supplementation is now widely available. It has anti-inflammatory activity that has been proven in cell cultures, rodents, and healthy human volunteers. Cell cultures studies have demonstrated that EPA inhibits productions of TNF- $\alpha$, IL-1b, IL- 6 and IL-8 by monocytes, macrophage, and endothelial cells. Hence, fish oil feeding inhibits rodent macrophage production of TNF- $\alpha$, IL-1b and IL- 6 while in human, fish oil supplementation also reduces the production of TNF, or IL-1 or IL- 6 by mononuclear cells. ${ }^{13}$

Anti-inflammatory property of EPA is due to its effect in altering the expression of genes encoding the cytokines. It is suggested that EPA modifies activities of transcription factors, such as NF- $\kappa \mathrm{B}$ and/or peroxisome proliferator activated receptor (PPAR)- $\gamma$. Eicosapentaenoic acid inhibits phosphorylation of I $\mathrm{KB}$, which then decreases the activation of NF- $\kappa B$ and alter the cytokine gene expressions. EPA may also bind to and activate PPAR- $\gamma$, which is an anti-inflammatory mediators transcription factor, enhancing its activity. ${ }^{13}$

Anti-inflammatory activity of EPA may have beneficial effects on cancer cachexia. In addition to this anti-inflamatory activity, EPA also has a potential mechanism to block other cachexia underlying factors (Fig. 2).

Proteolysis activity in cachexia can be inhibited by EPA. The decreased production of TNF- $\alpha$ and IL- 6 restricts their action on IкB $\alpha$. In addition, EPA attenuates the action of PIF on I $\kappa \mathrm{B} \alpha$. This decreased activity of I $\mathrm{B} \alpha$ restricts the activation of NF- $\kappa B$ that will then prohibits the action of ligase. This decreased activity also prevents the proteolysis of muscle fibers protein. ${ }^{14}$
Lipid depletion in cachexia can also be blocked by EPA. The LMF action in adipose tissue is attenuated by EPA. Eicosapentaenoic acid increases Gi expression while reducing Gs expression. This increase in Gi inhibits adenylate cyclase and, therefore, inactivates triglyceride lipase and also prevents lipolysis (Fig. 2). ${ }^{15}$

\section{Clinical Studies}

The beneficial effect of EPA for treatment of cachexia remains inconclusive in many clinical studies. A double blind placebo controlled trial performed by Fearon et al. ${ }^{14}$ showed no statistically significant benefit of EPA as a treatment for cancer cachexia. However, in that randomized control trial, patients treated with $2 \mathrm{~g}$ of EPA gained about $1 \mathrm{~kg}$ of body weight compared with those receiving placebo. These patients also showed modest improvement in physical function compared to those receiving placebo. In this trial, instead of $2 \mathrm{~g}$ of EPA, some patients were randomly assigned to receive $4 \mathrm{~g}$ of EPA. However, patients receiving $4 \mathrm{~g}$ of EPA showed no benefit, or rather showed deteriorating effect. It is suggested that the higher EPA doses may create adverse effects that impair gastrointestinal function, thus decreasing food intake. ${ }^{16}$ Another trial by Fearon et al..$^{14}$ suggested that EPA supplementation and identical supplementation without EPA are equally effective in maintaining body weight. However, in post-hoc analysis, only patients receiving EPA supplementation gained body weight and lean tissue mass as was as achieving improvement in quality of life. ${ }^{14}$

Experimental studies by Murphy et al. ${ }^{17}$ suggested that the supplementation of $2.5 \mathrm{~g}$ of EPA and DHA significally increases the efficacy of chemotherapy compared to placebo among patients with lung cancer. Another study in lung cancer patients showed that patients received $2.02 \mathrm{~g} \mathrm{EPA}+0.92 \mathrm{DHA}$ had better body weight and fat free mass maintenance compared to control group. ${ }^{18}$ Supplementation with fish oil also improved quality of life, performance status, and physical activity compared to placebo. ${ }^{19}$

It is concluded that eicosapentaenoic acid shows potential benefits in treatment of cancer cachexia. Further studies are required to determine the role of EPA as an adjuvant for cancer cachexia. 


\section{References}

1. Tisdale MJ. Mechanisms of cancer cachexia. Physiol Rev. 2009;89(2):381-410.

2. Kumar R, Abbas A, DeLancey, Malone E. Robbin and Cotran pathologic basis of disease. $8^{\text {th }} \mathrm{ed}$. Philadelphia: Elsevier; 2010.

3. Donohoe CL, Ryan AM, Reynolds JV. Cancer cachexia: mechanisms and clinical implications. Gastroenterol Res Pract. 2011;2011:1-13.

4. Gordon JN, Green SR, Goggin PM. Cancer cachexia. QJM. 2005;98(11):779-88.

5. Topkan E, Yavuz AA, Ozyilkan O. Cancer cachexia: pathophysiologic aspects and treatment options. Asian Pac J Cancer Prev. 2007;8(3):445-51.

6. 1st Abbott international conference for cancer nutrition therapy. 1st Abbott international conference for cancer nutrition therapy; April 12-14; Istanbul Turkey: Abbott Nutrition; 2010.

7. Radbruch L, Elsner F, Trottenberg P, Strasser F, Baracos V, Fearon K. Clinical practice guidelines on cancer cachexia in advanced cancer patients with a focus on refractory cachexia. Aachen: Department of Palliative Medicinen/European Palliative Care Research Collaborative; 2010.

8. Stewart GD, Skipworth RJ, Fearon KC. Cancer cachexia and fatigue. Clin Med. 2006;6(2):1403.

9. Coletti D, Belli L, Adamo S. Cachexia: Novel perspectives for an old syndrome. Basic Appl Myol. 2006;16(5\&6):131-9.

10. Tisdale MJ. Pathogenesis of cancer cachexia. J Support Oncol. 2003;1(3):159-68.

11. Lenhard RE, Ostieen RT, Gansleer T. Clinical oncology. $1^{\text {st }}$ ed. Atlanta: American Cancer Society; 2001.

12. Grunberg SM. Chemotherapy-induced nausea and vomiting: prevention, detection, and treatment-how are we doing. J Support Oncol. 2004;2(Suppl 1):S1-12.
13. Calder PC. Polyunsaturated fatty acids and inflammatory processes: new twists in an old tale. Biochimie. 2009;91(6):791-5.

14. Fearon KCH, Von Meyenfeldt MF, Moses AGW, Van Geenen R, Roy A, Gouma DJ, et al. Effect of a protein and energy dense N-3 fatty acid enriched oral supplement on loss of weight and lean tissue in cancer cachexia: a randomised double blind trial. Gut. 2003;52(10):1479-86.

15. Islam-Ali B, Khan S, Price S, Tisdale MJ. Modulation of adipocyte G-protein expression in cancer cachexia by a lipid-mobilizing factor (LMF). Br J Cancer. 2001;85(5):758-63.

16. Fearon KC, Barber MD, Moses AG, Ahmedzai $\mathrm{SH}$, Taylor GS, Tisdale MJ, et al. Doubleblind, placebo-controlled, randomized study of eicosapentaenoic acid diester in patients with cancer cachexia. J Clin Oncol. 2006;24(21):3401-7.

17. Murphy RA, Mourtzakis M, Chu QS, Baracos VE, Reiman T, Mazurak VC. Supplementation with fish oil increases first-line chemotherapy efficacy in patients with advanced nonsmall cell lung cancer. Cancer. 2011;117(16):377480.

18. van der Meij B, Langius J, Smit E, Spreeuwenberg $\mathrm{M}$, von Blomberg B, Heijboer A, et al. Oral nutritional supplements containing (n3) polyunsaturat ed fatty acids affect the nutritional status of patients with stage III nonsmall cell lung cancer during multimodality treatment. J Nutr. 2010;140(10):1774-80.

19. van der Meij BS, Langius JAE, Spreeuwenberg MD, Slootmaker SM, Paul MA, Smit EF, et al. Oral nutritional supplements containing n-3 polyunsaturated fatty acids affect quality of life and functional status in lung cancer patients during multimodality treatment: an RCT. Eur J Clin Nutr. 2012;66(3):399-404. 